DOI: http://dx.doi.org/10.18524/1810-4215.2017.30.114335

\title{
SYNTHESIS OF MAGNETIZED NUCLEI AT SUPERNOVA EXPLOSION
}

\author{
V.N.Kondratyev $^{1,2}$, U.M.Nurtayeva ${ }^{2,4}$, A.Zh.Zhomartova ${ }^{2,4}$, T.V.Mishenina ${ }^{3}$ \\ ${ }^{1}$ Physics Department, Taras Shevchenko National University of Kyiv, \\ 03022-UA Kyiv, Ukraine,vkondrat@univ.kiev.ua \\ ${ }^{2}$ BLTP, JINR, 141980-RU Dubna, Russia \\ ${ }^{3}$ Astronomical Observatory, Odessa National University, \\ 65014-UA Odessa, Ukraine \\ ${ }^{4}$ Department of Experimental Methods in Nuclear Physics, MEPhI NRNU, \\ Moscow, Russia
}

\begin{abstract}
Influence of magnetorotational instabilities in astrophysical plasma at supernova explosion on synthesis of chemical elements is investigated. At field strength less than 10 teratesla nuclear magnetic susceptibility exhibits linear regime with enhanced nuclear binding energy for open shell nuclei. Effects of ultra-strong nuclear magnetization are demonstrated to enhance the portion of titanium product. The relation to an excess of titanium isotopes revealed from the Integral mission data and galactic chemical evolution is discussed.
\end{abstract}

Keywords: Stars: supernovae, magnetic field. - Nucleosynthesis: abundances, the Galaxy chemical evolution.

\section{Introduction}

Ultrastrong magnetic fields exceeding teratesla (TT) arise at core-collapse supernova (SN) explosion (Kondratyev 2014), in magnetar crusts and heavy ion collisions. Nuclides produced in such processes contain an information on matter structure and explosion mechanisms. In this work we analyze effect of relatively weak magnetic field in nuclear structure and discuss possibilities for using radionuclides to probe internal regions of respective sites.

Interaction of nucleon magnetic moment $m_{\mathrm{N}}$ with a field $H$ leads to a shift of energy levels $\Delta=m_{\mathrm{N}} H$. Dramatic change in nuclear structure corresponds to conditions of level crossing. The nuclear level spacing $\Delta E \sim 1 \mathrm{MeV}$ gives respective field strength scale $\Delta H_{\text {cross }} \sim \Delta E / \mu_{\mathrm{N}} \sim 10 \mathrm{TT}$. Here $\mu_{\mathrm{N}}$ denotes the nuclear magneton. In a case of smaller strengths $H<10 \mathrm{TT}$ one can use a linear approximation, cf., (Kondratyev \& Korovina, 2015). In next section we demonstrate that magnetic susceptibility at a field strength of teratesla exceeds significantly respective ground state $g$-factor corresponding to vanishing magnetic induction. Effect of magnetic field in nuclear composition is considered in sect. 3 . Conclusions are in sect. 4.

\section{Structure of magnetized atomic nuclei}

The single-particle (sp) Hamiltonian $\mathrm{H}_{\alpha}$ for nuclei within non-relativistic approximation and a linear limit in a weak magnetic field $\mathbf{H}$ reads

$$
\mathrm{H}_{\alpha}=\mathrm{H}_{\alpha}{ }^{0}-\left(g_{\alpha}{ }^{\mathrm{o}} \mathbf{l}-g_{\alpha} \mathbf{s}\right) \omega_{\mathrm{L}}
$$

for protons $\alpha=p$ and neutrons $\alpha=n$ with well known $\mathrm{g}$ factors $g_{\alpha}$. Here $\mathrm{H}_{\alpha}{ }^{0}$ represents the sp Hamiltonian for isolated nuclei, $\mathbf{l}$ and $\mathbf{s}$ denote operators of the orbital momentum and spin. An interaction of nucleon dipole magnetic moment with magnetic field is represented by terms containing the vector $\omega_{\mathrm{L}}=\mu_{\mathrm{N}} H$.

The nuclear binding energy $\Delta B=\sum_{\text {occ }} e_{\mathrm{i}}$ is given as a sum over occupied levels $\mathrm{i}$ of $\mathrm{sp}$ eigen energies $e_{\mathrm{i}}$. By making use of angular momentum representation for spherical nuclei one can write the change of binding energy in magnetic field as

$$
\begin{aligned}
& \Delta B_{\alpha}=\kappa_{\alpha} \omega_{\mathrm{L}}, \kappa_{\alpha}=\Sigma_{\mathrm{i}-\mathrm{occ}} \kappa_{\alpha}^{\mathrm{i}} \\
& \kappa_{\alpha}^{\mathrm{i}}=\Sigma_{m, s}|<l m, 1 / 2 s| j m_{j}>\left.\right|^{2}\left(g_{\alpha}{ }^{\mathrm{o}} m+g_{\alpha} s\right),
\end{aligned}
$$

where $<l m,{ }^{\mathrm{o}_{1}} / 2 s \mid j m_{j}>$ represents the Clebsch-Gordan coefficient.

Thus, values $k$ are significantly different from nuclear $g$ factors corresponding to an interaction of nuclear magnetic moment in a ground state with magnetic field. Within the shell model nuclear magnetic moment is determined by a valent unpaired nucleons and associated nuclear g-factor is given by values of Eq. (2) and determined for states with maximum spin projection $m_{j}$ of positive and negative signs for protons and neutrons, see Tables 1.

Table 1. Susceptibility parameter $\kappa$ for iron shell closure region. Contributions of $\mathrm{Z}$ protons and $\mathrm{N}$ neutrons are indicated by $\kappa_{\mathrm{p}}$ and $\kappa_{\mathrm{n}}$.

\begin{tabular}{|c|c|c|c|}
\hline$Z$ & \multicolumn{1}{|l}{$\kappa_{\mathrm{p}}$} & \multicolumn{1}{l}{$\kappa_{\mathrm{n}}$} \\
\hline 20 & 0.00 & 20 & 0.00 \\
\hline 21 & 5.793 & 27 & 1.913 \\
\hline 22 & 9.931 & 26 & 3.279 \\
\hline 23 & 12.414 & 25 & 4.099 \\
\hline 24 & 13.241 & 24 & 4.373 \\
\hline 25 & 12.414 & 23 & 4.099 \\
\hline 26 & 9.931 & 22 & 3.279 \\
\hline 27 & 5.793 & 21 & 1.913 \\
\hline 28 & 0.00 & 28 & 0.00 \\
\hline
\end{tabular}

Evidently, such strong nuclear magnetization leads to an increase of binding energy. As is seen from Eqs. (3) and in 
Table 1 open shell nuclei experience maximum of such extra binding. For protons effect is about 3 times larger as compared to neutrons. Consequently, composition of stable nuclei in magnetic field is modified. For instance, for often considered isobars ${ }^{44} \mathrm{Z}$, e.g., ${ }^{44} \mathrm{Sc}$ and ${ }^{44} \mathrm{Ti}$, from Table 1 we have $\left(k_{\mathrm{Ti}}-k_{\mathrm{Sc}}\right)=3.32$ and at a field strength $\mathrm{H}=2.5 \mathrm{TT}$ the nucleus ${ }^{44} \mathrm{Ti}$ is stronger bound than ${ }^{44} \mathrm{Sc}$. It is worthy to recall here that ${ }^{44} \mathrm{Ti}$ becomes the most tightly bound nucleus at $\mathrm{H}=20 \mathrm{TT}$, see (Kondratyev, 2014; Kondratyev \& Korovina, 2015). For a weak field limit we notice similar results obtained from consideration within the covariant density functional theory (Pena Arteaga et al., 2011) and shell correction approach (Kondratyev \& Korovina, 2015) which exclude an interaction of total magnetic moment of a nucleus with magnetic field.

\section{Synthesis of magnetized nuclei}

Such a magnetic medication of nuclear structure affects nuclide production at respective explosion. Nuclear statistical equilibrium (NSE) approach gives useful tool used very successfully for description and analysis of abundances of iron group and nearby nuclides for over half a century (Woosley, Heger \& Weaver, 2002). At such conditions nuclide yield is determined mainly by the binding energy of corresponding atomic nuclei. The magnetic effects in the NSE were considered by Kondratyev (2014, and refs. therein). Recall that at temperatures $\left(T \leq 10^{9.5} \mathrm{~K}\right)$ and field strengths $(H \geq 0.1 \mathrm{TT})$, the magnetic field dependence of relative output value $y=Y(H) / Y(0)$ is determined by a change in the binding energy of nuclei in a field $\mathrm{H}$ and can be written in the following form

$$
y=\exp \{\Delta B / k T\} \approx \exp \left\{\kappa \omega_{\mathrm{L}} / k T\right\} .
$$

We used here Eq. (2).

We consider examples of iron group and titanium. Such a choice of double magic and anti-magic nuclei at vanishing magnetization gives a clear picture of magnetic effects in the formation of chemical elements and fundamental conclusions about transmutation and synthesis of nuclei in ultramagnetized plasma.

It is worthy to recall in this regards there is a significant excess as compared to model predictions of $\mathrm{Ti}$ nuclei abundance obtained in direct observations of SN remnants (Kondratyev \& Korovina, 2015). Furthermore, there is the difference between $\mathrm{Ti}$ abundance revealed from galactic chemical evolution (GCE) predictions and the Ti content observations in stars (Kondratyev\&Mishenina 2016). Till now, none of the existing GCE codes does not describe the observational trend of $[\mathrm{Ti} / \mathrm{Fe}]$ vs. $[\mathrm{Fe} / \mathrm{H}]$. This is primarily due to the difficulties in calculating of the present theoretical stellar yields from massive supernovas that are the main source for titanium probably. This means that improved of the core collapse supernovae nucleosynthesis predictions from realistic models are required. Among others, this needs a detailed study of the role of rotation and magnetic fields (Mishenina et al., 2017).
As is seen from Eqs. (2), (3) and Table 1 an excess on order of magnitude corresponds to field strength of several TT. As is evident from Eqs. (2) maximum of magnetic susceptibility $\kappa$ corresponds to half filled shells. In case of iron closure $1 \mathrm{f}_{7 / 2}$ shell such a condition is met at $Z=N=24$. Then significant value of parameter $\kappa_{\mathrm{Cr}}=17.33$ implies strong magnetic enhancement in production of ${ }^{48} \mathrm{Cr}$ nuclde. The radioactive decay chain ${ }^{48} \mathrm{Cr} \rightarrow{ }^{48} \mathrm{~V} \rightarrow{ }^{48} \mathrm{Ti}$ gives rise to an excess of major titanium isotope.

\section{Conclusion}

We employ arguments of nuclear statistical equilibrium to study nucleosynthesis at magnetorotational supernova explosion. As is shown binding energy of nuclei with open shells increases at relatively weak fields corresponding to linear nuclear magnetic response Such a property suggests possible mechanism of additional titanium enrichment when taking into account the increased yield of anti-magic nuclides in ultramagnetized astrophysical plasma. As is seen on an example of the radioactive isotope ${ }^{44} \mathrm{Ti}$ the direct observational data, see sect. 1, confirm such an enrichment which can be understood in terms of magnetic effects. The resulting enrichments of M44 isobars are collaborated with observational data (Kondratyev, 2014; Magkotsios, 2010). The proton magnetic reactivity dominates in a change of binding energy, see Eq. (2). Noticeable increase in production of ${ }^{48} \mathrm{Cr}$ leads to increasing volume of stable titanium isotopes, as well. At the same time a yield of iron and calcium isotopes can be expected unchanged because of the proton shell closure, see Eqs. (2), (3) and Table 1.

Acknowledgements. This work is supported in part by Integral scientific data center and by the Swiss National Science Foundation (SCOPES project No. IZ73Z0152485).

\section{References}

Kondratyev V.N.: 2014, EPJ A, 50, 7.

Kondratyev V.N., Korovina Yu.V.: 2015, JETP L, 102, 131.

Kondratyev V.N., Mishenina T.V.: 2016, OAP, 29, 60.

Magkotsios G., Timmes F.X., Hungerford A.L. et al.: 2010, ApJS, 191, 66.

Mishenina T., Pignatati M., Côté B. et al.:2017, MNRAS, 469, 4378.

Peña Arteaga D., Grasso M., Khan E., Ring P.: 2011, Phys. Rev. C, 84, id.045806.

Woosley S.E., Heger A., Weaver T.A.: 2002, Rev. Mod. Phys., 74, 1015. 